\title{
BEM-ESTAR NA PRODUÇÃO DE FRANGO DE CORTE EM DIFERENTES SISTEMAS DE CRIAÇÃO
}

\section{AÉRICA C. NAZARENO ${ }^{1}$, HÉLITON PANDORFI ${ }^{2}$, CRISTIANE GUISELINI ${ }^{3}$, RICARDO B. $^{2}$ VIGODERIS ${ }^{4}$, ELVIRA M. R. PEDROSA ${ }^{5}$}

RESUMO: Esta pesquisa foi conduzida com o objetivo de avaliar diferentes sistemas de criação para frangos de corte, caracterizando o ambiente térmico, os parâmetros fisiológicos e o comportamento animal. O experimento foi realizado no decorrer de um ciclo produtivo de 42 dias, no município de Carpina, Estado de Pernambuco, na Estação Experimental de Pequenos Animais da Universidade Federal Rural de Pernambuco (UFRPE). A pesquisa foi desenvolvida em um módulo de produção dividido em 15 boxes, com 10 aves por Box, e densidade de 10 aves por $\mathrm{m}^{2}$, totalizando 150 aves, submetidas a três sistemas de criação: semiconfinado com $3 \mathrm{~m}^{2}$ por ave de área de piquete (SC 3), semiconfinado com $6 \mathrm{~m}^{2}$ por ave de área de piquete (SC 6) e confinamento total (CONF). O delineamento experimental para análise geral dos dados foi inteiramente casualizado (DIC) em parcelas subdivididas, sendo as semanas alocadas nas parcelas, e os sistemas de criação (SC 3, SC 6, CONF e EXT), nas subparcelas, com as médias comparadas pelo teste de Tukey. As variáveis meteorológicas, índices de conforto e os parâmetros fisiológicos apontam o sistema de criação SC 3 como aquele que permitiu melhor acondicionamento térmico natural às aves, apresentando valores médios da ordem de $25,4{ }^{\circ} \mathrm{C} ; 69,9 \mathrm{~kJ} \mathrm{~kg}^{-1} ; 75,7 ; 65,12$ movimentos $\min ^{-1}$ e $41,92{ }^{\circ} \mathrm{C}$ para temperatura de bulbo seco, entalpia, índice de temperatura de globo e umidade, frequência respiratória e temperatura cloacal, respectivamente. As aves submetidas ao sistema de criação SC 3 foram as que tiveram melhor oportunidade de expressar seus comportamentos naturais e de explorar o ambiente externo ao módulo de criação, potencializando o bem-estar animal.

PALAVRAS-CHAVE: avicultura, conforto térmico, comportamento, instalações agrícolas.

\section{ANIMAL WELFARE IN DIFFERENT HOUSING SYSTEMS OF BROILER PRODUCTION}

\begin{abstract}
This research was carried out to evaluate different housing systems for broiler chickens production, characterizing the thermal environment, physiological parameters and animal behavior. The experiment was conducted in a production cycle of 42 days, in the city of Carpina, state of Pernambuco, Brazil, at the Experimental Station for Small Animals of the Federal Rural University of Pernambuco (UFRPE). The survey was developed in production module divided into 15 boxes with 10 birds per box and density of the 10 birds per $\mathrm{m}^{2}$, totaling 150 birds, submitted to the three housing systems: semiconfined with $3 \mathrm{~m}^{2}$ per broiler of paddock area (SC 3), semi-confined with $6 \mathrm{~m}^{2}$ per broiler of paddock area (SC 6) and total confinement (CONF). The experimental design for data general analysis was completely randomized (CRD), in split plots, being the weeks allocated in the plots and the accommodation systems (SC 3, SC 6, CONF and EXT) in the sub-plots with the averages compared through the Tukey test. The meteorological variables, comfort indexes and physiological parameters indicated the semi-confined system of accommodation SC 3 as one that allowed better natural conditioning heat to the birds, presenting the average values of $25.4^{\circ} \mathrm{C}, 69.9 \mathrm{~kJ} \mathrm{~kg}^{-1}, 75.7,65.12$ movements $\min ^{-1}$ and $41.92^{\circ} \mathrm{C}$ for dry bulb temperature, enthalpy, black globe humidity index, respiratory rate and cloacal temperature, respectively. The birds subjected to the semi-confined system SC 3 were those that had better opportunity to express their natural behavior and explore the external environment to the module of production, leveraging the animal welfare.
\end{abstract}

KEYWORDS: poultry production, thermal comfort, animal behavior, housing systems.

\footnotetext{
${ }^{1}$ Eng ${ }^{-}$Agrônomo, Me. Eng. Agrícola, PPG em Engenharia Agrícola - UFRPE.

${ }^{2}$ Eng $^{\mathrm{O}}$ Agrônomo, Professor Adjunto, Departamento de Tecnologia Rural - UFRPE, pandorfi@dtr.ufrpe.br.

${ }^{3}$ Eng $^{0}$ Agrônomo, Professor Adjunto, Departamento de Tecnologia Rural - UFRPE.

${ }^{4}$ Eng $^{\mathrm{O}}$ Agrícola, Professor Adjunto, Unidade Acadêmica de Garanhuns - UFRPE.

${ }^{5}$ Eng $^{\mathrm{o}}$ Agrônomo, Professor Associado, Departamento de Tecnologia Rural - UFRPE.

Recebido pelo Conselho Editorial em: 20-4-2010

Aprovado pelo Conselho Editorial em: 18-11-2010
} 


\section{INTRODUÇÃO}

Os avanços da genética, nutrição e manejo fizeram da produção brasileira de frangos de corte um grande complexo agroindustrial, capaz de expandir as fronteiras do mercado externo aos patamares de maior destaque no cenário mundial. Estima-se que, em 2010, a produção de carne de frango alcance 11,44 milhões de toneladas e consumo de 42,21 kg habitante ano ${ }^{-1}$ (ANUALPEC, 2009). Por outro lado, os principais importadores de proteína animal possuem normativas específicas referentes às boas práticas pautadas no bem-estar, acompanhadas por forte tendência dos consumidores em adquirir produtos desenvolvidos a partir de princípios éticos e com qualidade garantida desde a origem, ou seja, com a observação de todas as etapas dos sistemas de produção.

O bem-estar animal pode ser considerado uma demanda para que um sistema de produção seja defensável eticamente e aceitável socialmente, pois segundo VERCOE et al. (2000) e FRASER (2001), os consumidores desejam comer carne com atributos diferenciados, isto é, carne oriunda de animais que foram criados, tratados e abatidos em sistemas que promovam o seu bem-estar, e que sejam ambientalmente corretos.

A quantificação do ambiente térmico ao qual um animal está submetido não é suficiente para se obterem as reais necessidades de bem-estar dos animais, sendo que o ambiente de criação influencia diretamente em sua expressão comportamental. Ajustes de comportamento podem ocorrer rapidamente e a um custo menor do que os ajustes fisiológicos, beneficiando a produção (PEREIRA \& NÄÄS, 2005; PEREIRA \& NÄÄS, 2008).

O sistema de criação semi-intensivo é considerado como uma alternativa que permite livre acesso das aves às áreas de pastejo, resultando em diferenças particulares na expressão de seus comportamentos naturais, bem-estar e na qualidade do produto final (BLOKHUIS et al., 2000). O estudo do comportamento assume papel importante dentro da produção animal, uma vez que, para racionalizar os métodos de criação, têm-se desenvolvido técnicas de manejo, alimentação e instalações que interferem no comportamento animal (TAKAHASHI et al., 2006; PEREIRA et al., 2006; ALVES et al., 2007; TUYTTENS et al., 2008).

A criação de frangos de corte tipo colonial no Brasil utiliza uma área de três metros quadrados de piquete por ave, a criação pode ser intensiva até os 28 dias de idade e extensiva (com acesso a piquete), após esse período (TAKAHASHI et. al., 2006). Com este fundamento, foi então que se utilizou o sistema de criação semiconfinado com $3 \mathrm{~m}^{2}$ por ave de área de piquete e o dobro desta área para o semiconfinado com $6 \mathrm{~m}^{2}$ por ave de área de piquete, tratando-se de um manejo alternativo para frangos de corte de linhagem de crescimento rápido, visando à maior liberdade de movimento e bem-estar às aves.

Nesse contexto, objetivou-se com esta pesquisa avaliar diferentes sistemas de criação para frangos de corte, caracterizando o ambiente térmico, os parâmetros fisiológicos e o comportamento das aves em resposta ao ambiente de criação.

\section{MATERIAL E MÉTODOS}

O experimento foi realizado na Estação Experimental de Pequenos Animais da Universidade Federal Rural de Pernambuco (UFRPE), no município de Carpina, Estado de Pernambuco (latitude de $7,85^{\circ} \mathrm{S}$, longitude de $35,24^{\circ} \mathrm{W}$ e altitude de $180 \mathrm{~m}$ ). O clima da região é caracterizado como megatérmico (As'), com precipitação de inverno e estação seca do verão até o outono, segundo classificação de Köeppen (PEREIRA et al., 2002). Foram utilizados 150 pintos de corte, sexo misto, linhagem comercial Cobb 508, com ciclo de produção de 42 dias, de junho a julho de 2007.

A pesquisa foi desenvolvida em módulo de produção dividido em 15 boxes $\left(1 \mathrm{~m}^{2}\right)$, com 10 aves por box e densidade de 10 aves por $\mathrm{m}^{2}$, submetidas aos três sistemas de criação: semiconfinado com $3 \mathrm{~m}^{2}$ por ave de área de piquete (SC 3), semiconfinado com $6 \mathrm{~m}^{2}$ por ave de área de piquete (SC 6) e confinamento total (CONF), expostos ao acondicionamento térmico natural do local. O módulo de produção era de alvenaria, com piso de concreto, dividido por tela metálica, 
com presença de aberturas em cada um dos boxes, o que permitia o acesso das aves ao piquete, das 7 às $17 \mathrm{~h}$.

O trabalho foi dividido em duas etapas, em função da forma de avaliação dos dados: análise bioclimática e análise comportamental. A avaliação bioclimática foi realizada por meio do registro dos parâmetros fisiológicos, frequência respiratória (mov $\left.\mathrm{min}^{-1}\right)$ e temperatura cloacal $\left({ }^{\circ} \mathrm{C}\right.$ ), registrados em intervalos de 4 horas, às $9 ; 13$ e $17 \mathrm{~h}$, três vezes por semana. As aves foram selecionadas aleatoriamente, duas por repetição, em cada sistema de criação, totalizando trinta aves, identificadas por anilhas nas pernas de maneira que permanecessem fixas durante o dia selecionado.

O acompanhamento das variáveis meteorológicas, temperatura de bulbo seco $\left(\mathrm{Tbs} ;{ }^{\circ} \mathrm{C}\right)$, temperatura de bulbo úmido (Tbu; ${ }^{\circ} \mathrm{C}$ ) e temperatura de globo negro $\left(\mathrm{Tg} ;{ }^{\circ} \mathrm{C}\right)$, foi registrado diariamente nos boxes de criação e no ambiente externo, em intervalos de 2 horas, com registro às $7 ; 9 ; 11 ; 13 ; 15$ e $17 \mathrm{~h}$, o que permitiu a caracterização da eficiência térmica, pelos índices de temperatura de globo e umidade e entalpia. Para o registro dos dados ambientais, foram utilizados dataloggers modelo HOBO Pro HB8 (Onset Computer Corporation Bourne, MA, USA). Os sensores foram posicionados no centro geométrico dos boxes de produção, a 0,5 $\mathrm{m}$ do piso. No ambiente externo, os sensores foram instalados em abrigo meteorológico a 1,50 m de altura do solo.

Quando as aves atingiram 21 dias de idade, foi permitido o acesso aos piquetes nos sistemas de criação SC 3 e SC 6. Para a avaliação do comportamento das aves dentro e fora dos módulos de criação, foram escolhidas aleatoriamente 10 aves em cada sistema de criação, totalizando 20 aves para as observações, sendo duas aves por box, com cinco repetições. As aves foram identificadas com violeta genciana na cabeça, pescoço e asa, de maneira que permanecessem fixas para observação durante o dia selecionado, nos intervalos horários das 7 às $8 \mathrm{~h}, 10$ às $11 \mathrm{~h}, 13$ às $14 \mathrm{~h}$ e das 16 às $17 \mathrm{~h}$, duas vezes por semana, registrando-se os comportamentos a cada $5 \mathrm{~min}$, verificandose a frequência de acesso e tempo de permanência das aves no piquete.

O delineamento experimental para análise geral dos dados foi inteiramente casualizado com parcelas subdividas, sendo as semanas alocadas nas parcelas, e o sistema de criação (SC 3, SC 6, CONF) e o ambiente externo (EXT), nas subparcelas. Para a comparação das médias, utilizou-se o teste de Tukey $(\mathrm{P}<0,05)$. Os dados comportamentais foram avaliados pela análise das distribuições das frequências, que foram submetidas ao teste de qui-quadrado e probabilidade.

\section{RESULTADOS E DISCUSSÃO}

Observa-se, na Tabela 1, que os sistemas de criação e o ambiente externo apresentaram diferenças significativas $(\mathrm{P}<0,05)$ para os valores médios de temperatura de bulbo seco, verificando-se maior temperatura no sistema de criação CONF, em virtude da presença do maior número de animais permanentemente confinados, sem acesso ao piquete, ocasionado pelo maior aporte de energia gerado pelos animais, conforme descrito por FURTADO et al. (2003), seguido pelo SC 6, SC 3 e EXT.

$\mathrm{O}$ efeito foi significativo $(\mathrm{P}<0,05)$ para $\mathrm{Tg}$, verificando-se maior valor médio para o sistema de criação CONF, que diferiu dos sistemas de criação SC 6 e SC 3. O EXT apresentou o menor valor ao longo do período analisado, considerando-se que o estresse térmico ocasionado pelos componentes da energia radiante do ambiente é uma parcela significativa para a troca de calor sensível (PEREIRA et al., 2002; OWADA et al., 2007). O maior valor médio absoluto para UR foi registrado no sistema de criação CONF, e o menor, no EXT; no entanto, não diferiram estatisticamente, caracterizando a uniformidade da condição de entorno às instalações (Tabela 1). Os altos valores de umidade registrados foram decorrentes da época do ano, por se tratar de uma estação chuvosa, além do acúmulo de umidade na cama, proporcionada pelas excretas das aves, o que afetou diretamente as trocas térmicas entre os animais e o ambiente de criação. A faixa de UR considerada satisfatória para a melhor produção de frangos de corte está situada entre 50 e $70 \%$ (SARMENTO et al., 2005; OLIVEIRA et al., 2006). 
TABELA 1. Valores médios e desvio-padrão das variáveis ambientais para os sistemas de criação semiconfinado com $3 \mathrm{~m}^{2}$ por ave (SC3), semiconfinado com $6 \mathrm{~m}^{2}$ por ave (SC 6), confinamento total (CONF) e ambiente externo (EXT). Mean values and standard deviation of environmental variables for the production systems semi-confined with $3 \mathrm{~m}^{2}$ per broiler (SC 3), semi-confined with $6 \mathrm{~m}^{2}$ per broiler (SC 6), total confinement (CONF) and external environment (EXT).

\begin{tabular}{cccccc}
\hline $\begin{array}{c}\text { Sistema } \\
\text { de Criação }\end{array}$ & $\begin{array}{c}\text { Tbs } \\
\left({ }^{\circ} \mathrm{C}\right)\end{array}$ & $\begin{array}{c}\text { Tg } \\
\left({ }^{\circ} \mathrm{C}\right)\end{array}$ & $\begin{array}{c}\text { UR } \\
(\%)\end{array}$ & $\begin{array}{c}\mathrm{h} \\
\left(\mathrm{kJ} \mathrm{kg}^{-1}\right)\end{array}$ & ITGU \\
\hline SC 3 & $25,4 \mathrm{c} \pm 1,9$ & $26,6 \mathrm{~b} \pm 1,9$ & $81,5 \mathrm{a} \pm 4,5$ & $69,9 \mathrm{c} \pm 5,7$ & $75,7 \mathrm{c} \pm 2,4$ \\
SC 6 & $26,2 \mathrm{~b} \pm 2,0$ & $26,8 \mathrm{~b} \pm 1,9$ & $81,5 \mathrm{a} \pm 4,6$ & $70,8 \mathrm{~b} \pm 5,4$ & $76,0 \mathrm{~b} \pm 2,3$ \\
CONF & $26,8 \mathrm{a} \pm 2,0$ & $27,9 \mathrm{a} \pm 2,1$ & $81,8 \mathrm{a} \pm 5,1$ & $75,2 \mathrm{a} \pm 5,7$ & $77,5 \mathrm{a} \pm 2,5$ \\
EXT & $24,7 \mathrm{~d} \pm 1,9$ & $25,9 \mathrm{c} \pm 1,9$ & $80,5 \mathrm{a} \pm 3,5$ & $66,7 \mathrm{~d} \pm 6,0$ & $74,7 \mathrm{~d} \pm 2,5$ \\
\hline C.V. \% & 1,2 & 0,8 & 2,8 & 1,4 & 0,3 \\
Teste F & $121,1^{*}$ & $214,6^{*}$ & $8,0^{*}$ & $111,6^{*}$ & $258,2^{*}$ \\
\hline
\end{tabular}

Nas mesmas colunas, médias seguidas das mesmas letras não diferem entre si, a 5\% de probabilidade, pelo teste de Tukey. Tbs temperatura de bulbo seco; Tg - temperatura de globo negro; UR - umidade relativa; h - entalpia; e ITGU - índice de temperatura de globo e umidade; NS - não significativo, ${ }^{*}$ - significativo $(\mathrm{P}<0,05)$.

Nota-se que, entre os diferentes sistemas de criação, detectou-se maior valor de entalpia no sistema CONF, que diferiu dos sistemas de criação SC 6 e SC 3, que também diferiram entre si $(\mathrm{P}<0,05)$, apresentando valores da ordem de 75,2; 70,8 e 69,9 kJ kg ar seco-1 ${ }^{-1}$ respectivamente (TABELA 1). O sistema de criação que mais se aproximou do valor de entalpia adequado foi o SC 3, considerando-se o limite crítico superior para aves de $70 \mathrm{~kJ} \mathrm{~kg}^{-1}$, estabelecidos por BARBOSA FILHO et al. (2007). Com relação ao ITGU, deu-se efeito significativo $(\mathrm{P}<0,05)$ entre os sistemas de criação e o EXT, com maior valor médio no sistema de criação CONF, que diferiu dos sistemas de criação SC 6 e SC 3, sendo que o menor valor encontrado foi para o EXT (Tabela 1).

Considerando-se que o ITGU com valor de até 77 não influencia no desempenho das aves (SARMENTO et al., 2005), vê-se que apenas o sistema de criação CONF superou o limite recomendado, indicando que, em condições de campo, uma significativa parcela de sobreaquecimento advém do próprio calor gerado pelas aves, agravando mais a situação de desconforto térmico no interior das instalações.

Observa-se, na Tabela 2, que o sistema de criação SC 3 mostrou menor frequência respiratória $\left(65,1 \mathrm{mov}^{-1} \mathrm{~min}^{-1}\right)$, apresentando efeito significativo para os sistemas CONF e SC 6, decorrente do menor incremento térmico proporcionado pelas aves alojadas nesta condição, em que tiveram mais acesso à área de piquete, o que promoveu menor acréscimo (38\%) sob a taxa normal de frequência respiratória (MEDEIROS et al., 2005 a).

TABELA 2. Médias e desvio-padrão das variáveis fisiológicas nos sistemas de criação semiconfinado com $3 \mathrm{~m}^{2}$ por ave de área de piquete (SC 3), semiconfinado com $6 \mathrm{~m}^{2}$ por ave de área de piquete (SC 6) e confinamento total (CONF). Mean values and standard deviation of physiological variables for the production systems semiconfined with $3 \mathrm{~m}^{2}$ per broiler of paddock area (SC 3), semi-confined with $6 \mathrm{~m}^{2}$ per broiler of paddock area (SC 6), total confinement (CONF) and external environment.

\begin{tabular}{ccc}
\hline Sistema de Criação & Frequência Respiratória $\left({\left.\mathrm{mov} \mathrm{min}^{-1}\right)}\right)$ & Temperatura Cloacal $\left({ }^{\circ} \mathrm{C}\right)$ \\
\hline SC 3 & $65,1 \mathrm{~b} \pm 11,8$ & $41,8 \mathrm{c} \pm 0,3$ \\
SC 6 & $70,7 \mathrm{a} \pm 13,1$ & $41,9 \mathrm{~b} \pm 0,3$ \\
CONF & $70,8 \mathrm{a} \pm 11,9$ & $42,9 \mathrm{a} \pm 0,3$ \\
\hline C.V. \% & 2,9 & 0,1 \\
Teste F & $127,6^{*}$ & $184,7^{*}$ \\
\hline
\end{tabular}

Nas mesmas colunas, médias seguidas das mesmas letras não diferem entre si, a 5\% de probabilidade, pelo teste de Tukey.

* - significativo a $5 \%(\mathrm{P}<0,05)$. 
Quando a frequência respiratória está elevada, acima dos $40 \mathrm{mov} \min ^{-1}$ (MEDEIROS et al., 2005 b), admite-se que a temperatura do ar ultrapassa o limite crítico superior para as aves, pois o calor é armazenado no organismo, e o valor da temperatura corporal aumenta acima dos valores normais; esta resposta é decorrente do estímulo direto ao centro de calor no hipotálamo, que envia impulso ao sistema cardiorrespiratório, na tentativa de eliminar calor por evaporação por meio da respiração que, neste caso, apresenta aumento marcante em todos os sistemas de criação.

$\mathrm{O}$ aumento da frequência respiratória $\left(\mathrm{mov} \mathrm{min}^{-1}\right.$ ) nas aves submetidas aos sistemas de criação CONF e SC 6 foi causado pela impossibilidade e pela baixa demanda de acesso ao piquete, respectivamente, o que proporcionou incremento térmico no interior do módulo de criação, tendo como resposta direta o aumento de $44 \%$ na frequência respiratória das aves, com valor médio de $70,8 \mathrm{mov} \mathrm{min}^{-1}$ e $70,7 \mathrm{mov} \mathrm{min}^{-1}$, respectivamente, não indicando diferenças significativas entre ambos.

Outro ponto importante é que a frequência respiratória é influenciada pela idade da ave, pois quanto maior a idade maior também o número de vezes que a ave inspira ar por minuto, frente à sensibilidade térmica que as aves apresentam em idades mais avançadas (SILVA et al., 2007). O efeito isolado do sistema de criação para temperatura cloacal apresentou diferença significativa $(\mathrm{P}<0,05)$ entre os sistemas de criação, em virtude da dependência entre as médias e da variação observada nos desvios-padrão, em que a maior média ocorreu no sistema de criação CONF, e a menor, no SC 3; no entanto, as aves mantiveram as temperaturas cloacais dentro dos limites considerados normais, que variam entre 41 e $42{ }^{\circ} \mathrm{C}$, tanto no SC 3 quanto no SC 6 , de acordo com OLIVEIRA et al. (2006).

O sistema de criação CONF proporcionou maior valor médio de temperatura cloacal nas aves, comparado com os demais, devido à limitação de acesso ao piquete, pois, segundo SMITH \& OLIVER (1971) e YAHAV et al. (2000), a falta de espaço pode contribuir para o aumento da temperatura entre as aves, além de prejudicar ou impedir a troca de calor com o ambiente. Aves criadas no sistema semi-intensivo apresentam maior valor de peso corporal e menor valor de temperatura cloacal, taxa respiratória e hematócrito, demonstrando a influência positiva do sistema de criação nesses parâmetros e, consequentemente, no conforto e bem-estar das aves (SILVA et al., 2007).

Apresentam-se, na Tabela 3, a frequência e a percentagem de tempo em que os frangos de corte estiveram dentro e fora dos módulos de produção, verificando-se que o sistema de criação que apresentou maior frequência de aves dentro do módulo de criação (169 observações) foi o SC 6 , enquanto as aves que tiveram maior frequência ao piquete (105 observações) foi o SC 3; no entanto, não foi detectada diferença significativa entre os sistemas de criação.

TABELA 3. Frequência e percentagem média de permanência das aves dentro e fora do módulo de criação nos sistemas de criação semiconfinado com $3 \mathrm{~m}^{2}$ por ave de área de piquete (SC 3 ) e semiconfinado com $6 \mathrm{~m}^{2}$ por ave de área de piquete (SC 6). Frequency and mean percentage of time spent by birds inside and outside of creation module in production systems semi-confined with $3 \mathrm{~m}^{2}$ per broiler of paddock area (SC 3), semi-confined with $6 \mathrm{~m}^{2}$ per broiler of paddock area (SC 6).

\begin{tabular}{|c|c|c|c|}
\hline Frequência e Percentagem (\%) & SC 3 & SC 6 & Total \\
\hline Dentro & $\begin{array}{c}158 \\
30.00\end{array}$ & $\begin{array}{c}169 \\
3210\end{array}$ & $\begin{array}{c}327 \\
62.10\end{array}$ \\
\hline Fora & $\begin{array}{c}105 \\
20,00\end{array}$ & $\begin{array}{c}94 \\
17,90\end{array}$ & $\begin{array}{c}199 \\
37,90\end{array}$ \\
\hline $\begin{array}{c}\text { Qui-quadrado } \\
0837\end{array}$ & & & $\begin{array}{c}\text { Probabilidade } \\
0350\end{array}$ \\
\hline
\end{tabular}

Os motivos que provavelmente levaram as aves a permanecerem mais tempo dentro do módulo de criação no SC 6 foi a ausência de proteção às aves, que por se tratar de uma área maior 
(6 $\mathrm{m}^{2}$ ave $\mathrm{e}^{-1}$ ), a distância entre os limites do piquete e o módulo de criação poderia gerar uma situação de medo e frustração, reduzindo sua atividade de pastejo; consequentemente, notou-se maior preferência das aves no SC 3 em permanecerem mais tempo nos piquetes, destacando-se os limites mais estreitos e maior sensação de segurança aos animais (DAWKINS et al., 2004; DUNCAN, 2005).

Os motivos que provavelmente levaram as aves a permanecerem mais tempo dentro do módulo de criação no SC 6 foi a ausência de proteção às aves, que por se tratar de uma área maior $\left(6 \mathrm{~m}^{2}\right.$ ave $\left.^{-1}\right)$, a distância entre os limites do piquete e o módulo de criação poderia gerar uma situação de medo e frustração, reduzindo sua atividade de pastejo; consequentemente, notou-se maior preferência das aves no SC 3 em permanecerem mais tempo nos piquetes, destacando-se os limites mais estreitos e maior sensação de segurança aos animais (DAWKINS et al., 2004; DUNCAN, 2005).

A análise da percentagem de aves dentro e fora dos módulos de produção em relação à flutuação térmica diária, nos sistemas de criação SC 3 e SC 6 é apresentada nas Figuras 1; 2 e 3, em que, se consideraram os dias críticos para a condição de conforto das aves, estabelecidos com base no maior valor médio diário de entalpia, para $4^{\mathrm{a}}, 5^{\mathrm{a}}$ e $6^{\mathrm{a}}$ semanas do ciclo produtivo, de acordo com MOURA et al. (1997).

Nota-se, na Figura 1, que no sistema de criação SC 3, nos horários das 7; 11 e 17h; a percentagem de aves fora do módulo de criação foi maior do que dentro, apresentando valores da ordem de $68 ; 62$ e $60 \%$, respectivamente, coincidindo com os horários de temperaturas mais amenas e menor incidência de radiação, o que propiciou microclima favorável ao pastejo das aves.

No horário de temperatura mais elevada, às $13 \mathrm{~h}$, todas as aves preferiram ficar dentro do módulo de criação à sombra, pois nos piquetes elas ficariam expostas à radiação solar direta. Os frangos procuraram diminuir sua atividade de pastejo para tentar amenizar o estresse térmico, permanecendo dentro da instalação, evidenciando comportamentos de desconforto e aumento na ingestão de água. De acordo com MARCHINI et al. (2007), o horário das 13 às 15 h é considerado o mais desconfortante para as aves, o que promoveu aumento no consumo de até $50 \%$ do volume normal de água.

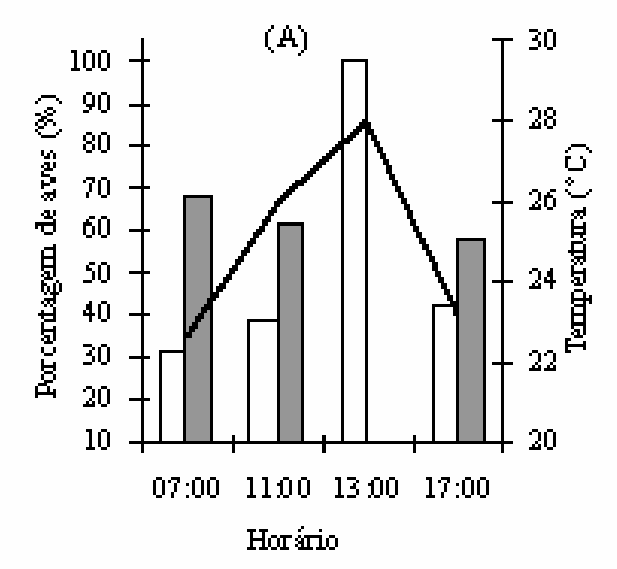

Dentro
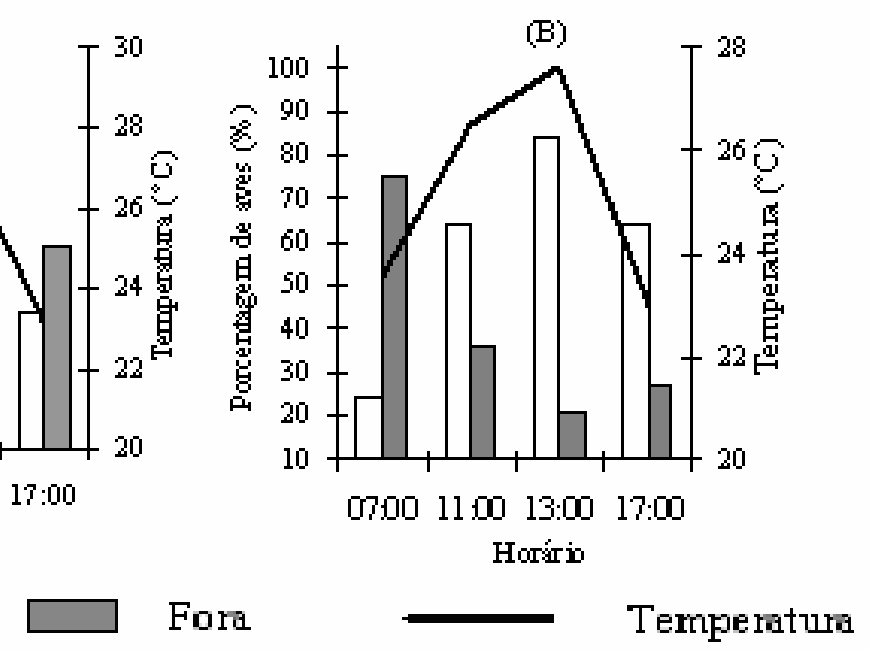

FIGURA 1. Avaliação da percentagem das aves dentro e fora do módulo de criação em relação à flutuação térmica diária para o dia crítico, na $4^{\underline{a}}$ semana do ciclo produtivo das aves, nos sistemas de criação SC 3 (A) e SC 6 (B). Evaluation of the percentage of birds inside and outside the creation module in relation to daily temperature fluctuation for the critical day in the $4^{\text {th }}$ week of the production in systems SC 3 (A) and SC 6 (B). 
No sistema de criação SC 6, o percentual de aves no piquete é maior apenas no horário das $7 \mathrm{~h}$ (75\%). Nos demais intervalos de observações, os animais tiveram preferência em permanecer no interior do módulo de criação (Figura 1B).

Verifica-se, na Figura 2, que o sistema de criação SC 3, nos horários das 7 e das 11h, a percentagem de aves fora do módulo de criação apresentou valores de 55 e $52 \%$, respectivamente. Observa-se redução expressiva no horário das $13 \mathrm{~h}$, em que as aves, na sua totalidade, estavam dentro do módulo de criação, evitando a exposição direta à radiação solar neste horário, retornando à área de piquete no intervalo do horário final, das 16 às $17 \mathrm{~h}$.

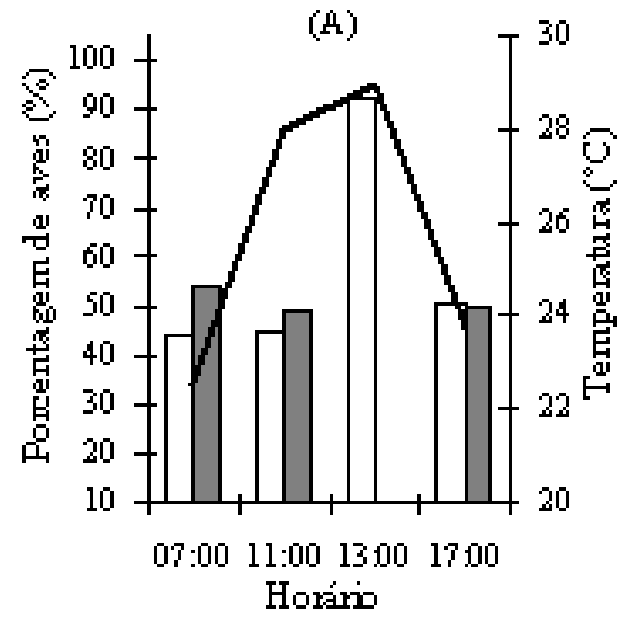

Dentro

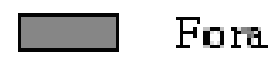

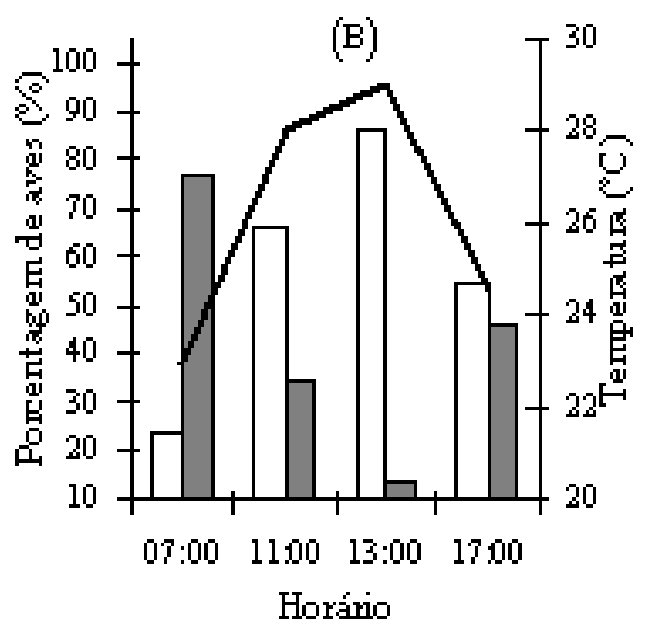

Temperatura

FIGURA 2. Avaliação da percentagem das aves dentro e fora do módulo de criação em relação à flutuação térmica diária para o dia crítico, na $5^{\mathrm{a}}$ semana do ciclo produtivo das aves, nos sistemas de criação SC 3 (A) e SC 6 (B). Evaluation of the percentage of birds inside and outside the creation module in relation to daily temperature fluctuation for critical day in the $5^{\text {th }}$ week of the production in systems SC 3 (A) and SC $6(B)$.

Durante todo o dia crítico para a $5^{\mathrm{a}}$ semana, o sistema de produção SC 6 apresentou maior percentagem de aves no piquete, comparativamente ao SC 3, para os intervalos horários estudados (Figura 2B). Um fator que exerce muita influência na expressão da atividade das aves é o horário, uma vez que todos os animais seguem um biorritmo, ligado principalmente à disponibilidade de radiação solar devido ao efeito da nebulosidade e à exposição a agentes estressores (PEREIRA et al., 2007).

Na Figura 3, nota-se que o percentual de aves fora do módulo de criação, na $6^{\mathbf{a}}$ semana do ciclo produtivo, foi inferior à percentagem de aves dentro, independentemente do sistema de criação e horário de observação.

O declínio no percentual de aves fora dos módulos de produção demonstra que o aumento na idade das aves dificulta sua locomoção devido ao acúmulo de massa corpórea, além do fato de se tratar de uma linhagem de crescimento rápido. À medida que as aves vão envelhecendo, o tempo em ócio aumenta (SEVEGNANI et al., 2005; TUYTTENS et al., 2008). 

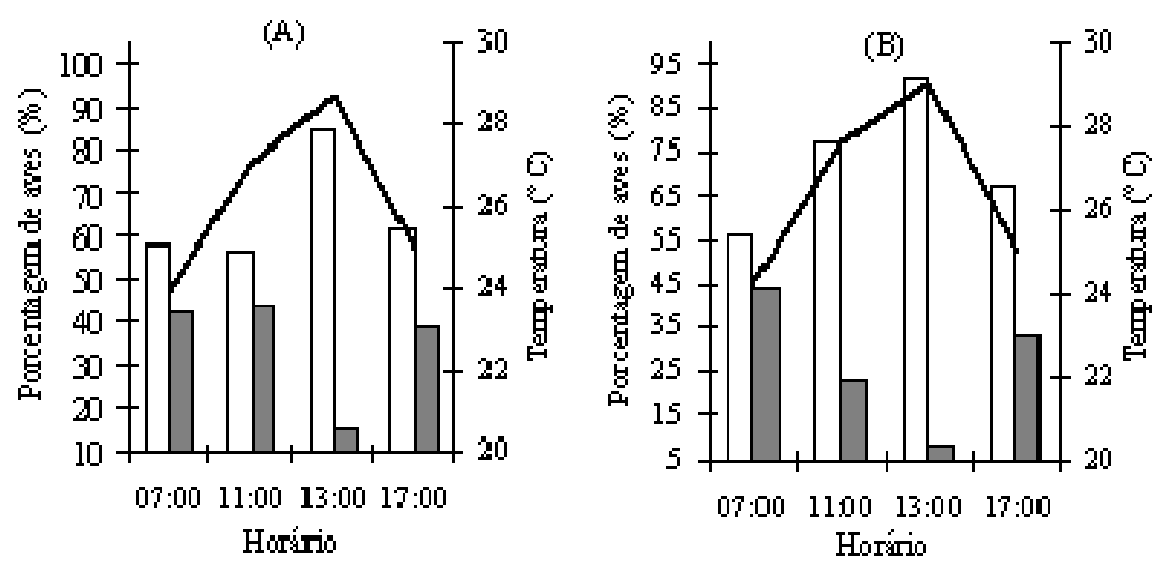

$\square$ Dentro $\square$ Fora

FIGURA 3. Avaliação da percentagem das aves dentro e fora do módulo de criação em relação à flutuação térmica diária para o dia crítico, na $6^{\mathrm{a}}$ semana do ciclo produtivo das aves, nos sistemas de criação SC 3 (A) e SC 6 (B). Evaluation of the percentage of birds inside and outside the creation module in relation to daily temperature fluctuation for critical day in the $6^{\text {th }}$ week of the production in systems SC 3 (A) and SC 6 (B).

De acordo com BESSEI (2006), o aumento da área de livre movimentação para o animal pode afetar sua saúde e seu bem-estar, uma vez que as aves permanecem com maior frequência no pasto e locomove-se mais, melhorando o seu bem-estar.

Um fato de grande relevância ao estímulo de acesso ao piquete seria a utilização de sombreamento artificial nos piquetes, para diminuir a exposição das aves à radiação solar direta, sendo que a utilização desse meio de climatização natural serviria como atrativo para as aves.

\section{CONCLUSÕES}

O sistema de criação semiconfinado, com $3 \mathrm{~m}^{2}$ por ave de área de piquete, foi o que melhor atendeu às exigências e à demanda das aves por um ambiente que lhe garanta maior liberdade de movimentação e conforto térmico ambiental, mostrando-se mais adequado às condições de bem-estar no alojamento das aves.

\section{REFERÊNCIAS}

ALVES, S.P.; SILVA, I.J.O.; PIEDADE, S.M.S. Avaliação do bem-estar de aves poedeiras comerciais: efeitos do sistema de criação e do ambiente bioclimático sobre o desempenho das aves e a qualidade de ovos. Revista Brasileira de Zootecnia, Viçosa-MG, v.36, n.5, p.1.388-1.394, 2007.

ANUALPEC 2009. Brasileiros devem consumir 83,8 kg de carnes, em 2009. Disponível em: $<$ http://www.rdmonline.com.br/index.php?n1=economia\&m=ver\&id_item=19133.htm>. Acesso em: 4 set. 2009.

BARBOSA FILHO, J.A.D.; SILVA, I..J.O.; SILVA, M.A.N.; SILVA, C.J.M. Avaliação dos comportamentos de aves poedeiras utilizando sequência de imagens. Engenharia Agrícola, Jaboticabal, v.27, n.1, p.93-99, 2007.

BESSEI, W. Welfare of broilers: a review. World's Poultry Science Journal, Cambrige, v.62, p.455466, 2006. 
BLOKHUIS, H.J.; EKKEL, E.D.; KORTE, S.M.; HOPSTER, H.; VAN REENEN, C.G. Farm animal welfare research in interaction with society. Veterinary Quarterly, Bilthoven, v.22, n.4, p.217-222, 2000.

DAWKINS, M.S.; DONNELLY, C.A.; JONES, T.A. Chicken welfare is influenced more by housing conditions than by stocking density. Nature, London, v.427, p.342-344, 2004.

DUNCAN, I.J.H. Science-based assessment of animal welfare: farm animals. Revue Scientifique et Technique (International Office of Epizootics), Paris, v.24, n.2, p.483-492, 2005.

FRASER, D. The "new perception" of animal agriculture: legless cows, featherless chickens and a need for genuine analysis. Journal of Animal Science, Champaign, v.79, n.3, p.634-641, 2001.

FURTADO, D.A.; AZEVEDO, P.V.; TINÔCO, I.F.F. Análise do conforto térmico em galpões avícolas com diferentes sistemas de acondicionamento. Revista Brasileira de Engenharia Agrícola e Ambiental, Campina Grande, v.7, n.3, p.559-564, 2003.

MARCHINI, C.F.P.; SILVA, P.L.; NASCIMENTO, M.R.B.M.; TAVARES, M. Frequência respiratória e temperatura cloacal em frangos de corte submetidos à temperatura ambiente cíclica elevada. Archives of Veterinary Science, Maringá, v.12, n,1, p.41-46, 2007.

MEDEIROS, C.M.; BAÊTA, F.C.; OLIVEIRA, R.F.M.; TINÔCO, I.F.F.; ALBINO, L.F.T.; CECON, P.R. Efeitos da temperatura, umidade relativa e velocidade do ar em frangos de corte. Engenharia na Agricultura, Viçosa-MG, v.13, n.4, p.277-286, 2005a.

MEDEIROS, C.M.; BAÊTA, F.C.; OLIVEIRA, R.F.M.; TINÔCO, I.F.F.; ALBINO, L.F.T.; CECON, P.R. Índice térmico ambiental de produtividade para frangos de corte. Revista Brasileira de Engenharia Agrícola e Ambiental, Campina Grande, v.9, n.4, p.660-665, 2005 b.

MOURA, D.J.; NÄÄS, I.A.; SILVA, I.J.O.; SEVEGNANI, K.B.; CORRIA, M.E. The use enthalpy as a thermal comfort index. Livestock Environment, St. Joseph, v.5, n.1, p.242-248, 1997.

OLIVEIRA, R.F.M.; DONZELE, J.L.; ABREU, M.L.T.; FERREIRA, R.A.; VAZ, R.G.M.V.; CELLA, P.S. Efeitos da temperatura e da umidade relativa sobre o desempenho e o rendimento de cortes nobres de frangos de corte de 1 a 49 dias de idade. Revista Brasileira de Zootecnia, ViçosaMG, v.35, n.3, p.797-803, 2006.

OLIVEIRA, G.A.; OLIVEIRA, R.F.M.; DONZELE, J.L.; CECON, P.R.; VAZ, R.G.M.V.; ORLANDO, U.A.D. Efeito da temperatura ambiente sobre o desempenho e as características de carcaça de frangos de corte dos 22 aos 42 dias. Revista Brasileira de Zootecnia, Viçosa-MG, v.35, n.4, p. 1398-1405, 2006.

OWADA, A.N.; NÄÄS, I.A.; MOURA, D.J.; BARACHO, M.S. Estimativa de bem-estar de frango de corte em função da concentração de amônia e grau de luminosidade no galpão de produção. Engenharia Agrícola, Jaboticabal, v.27, n.3, p.611-618, 2007.

PEREIRA, A.R.; ANGELOCCI, L.R.; SENTELHAS, P.C. Agrometeorologia: fundamentos e aplicações práticas. Guaíba: Agropecuária, 2002. 478 p.

PEREIRA, D.F.; CURTO, F.P.F. ; NÄÄS, I.A. Diferenças nos comportamentos individuais quanto à preferência de uso de locais de matrizes pesadas em função do ambiente térmico. Brazilian Journal of Veterinary Research and Animal Science, São Paulo, v.43, n.6, p.775-782, 2006.

PEREIRA, D.F.; NÄÄS, I.A. Estimating the thermoneutral zone for broiler breeders using behavioral analysis. Computers and electronics in agriculture, New York, v.62, n.1, p.2-7, 2008.

PEREIRA, D.F.; NÄÄS, I.A. Estimativa do conforto de matrizes de frango de corte baseada em análise do comportamento de preferência térmica. Engenharia Agrícola, Jaboticabal, v.25, n.2, p.315-321, 2005. 
PEREIRA, D.F.; SALGADO, D.D.; NÄÄS, I.A.; PENHA, N.L.J.; BIGHI, C.A. Efeitos da temperatura do ar, linhagem e período do dia nas frequências de ocorrências e tempos de expressão comportamental de matrizes pesadas. Engenharia Agrícola, Jaboticabal, v.27, n.3, p.596-610, 2007.

SARMENTO, L.G.V.; DANTAS, R.T.; TEIXEIRA, V.H.; FEREIRA, L. Efeito da pintura externa do telhado sobre o ambiente climático e o desempenho de frangos de corte. Agropecuária Técnica, Areia, v.26, n.2, p.117-122, 2005.

SEVEGNANI, K.B.; CAROR, I.W.; PANDORFI, H.; SILVA, I.J.O.; MOURA, D.J. Zootecnia de precisão: Análise de imagens no estudo do comportamento de frangos de corte em estresse térmico. Revista Brasileira de Engenharia Agrícola e Ambiental, Campina Grande, v.9, n.1, p.115-119, 2005.

SILVA, M.A.N.; BARBOSA FILHO, J.A.D.; SILVA, C.J.M.; ROSÁRIO, M.F.; SILVA, I.J.O.; COELHO, A.A.D.; SAVINO, V.J.M. Avaliação do estresse térmico em condição simulada de transporte de frangos de corte. Revista Brasileira de Zootecnia, Viçosa-MG, v.36, n.4, p.1.1261.130, 2007.

SMITH, A.J.; OLIVER, J. Some phisiological effects of high environmental temperatures on the laying hen. Poultry Science, Champaign, v.50, n.3, p.913-925, 1971.

TAKAHASHI, S.E.; MENDES, A.A.; SALDANHA, E.S.P.B.; PIZZOLANTE, C.C.; PELÍCIA, K.; GARCIA, R.G.; PAZ, I.C.L.A.; QUINTEIRO, R.R. Efeito do sistema de criação sobre o desempenho e rendimento de carcaça de frangos de corte tipo colonial. Revista Brasileira de Medicina Veterinária, Rio de Janeiro, v.58, n.4, p.123-132, 2006.

TUYTTENS, F.; HEYNDRICKX, M.; BOECK, M.; MOREELS, A.; NUFFEL, A.V.; POUCKE, E.V.; COILLIE, E.V.; DONGEN, S.V.; LENS, L. Broiler chicken health, welfare and fluctuating asymmetry in organic versus conventional production systems. Livestock Production Science, Amsterdam, v.113, n.1, p.123-132, 2008.

VERCOE, J.E.; FITZHUGH, H.A.; VON KAUFMANN, R. Livestock productions systems beyond Asian-Australian Journal of Animal Sciences, Seoul, v.13, p.411-419, 2000. Supplement

YAHAV, S.; SHINDER, D.; RAZPAKOVSKI, V.; RUSAL, M.; BAR, A. Lack of response of laying hens to relative humidity at high ambient temperature. British Poultry Science, London, v.41, p.660-663, 2000. 